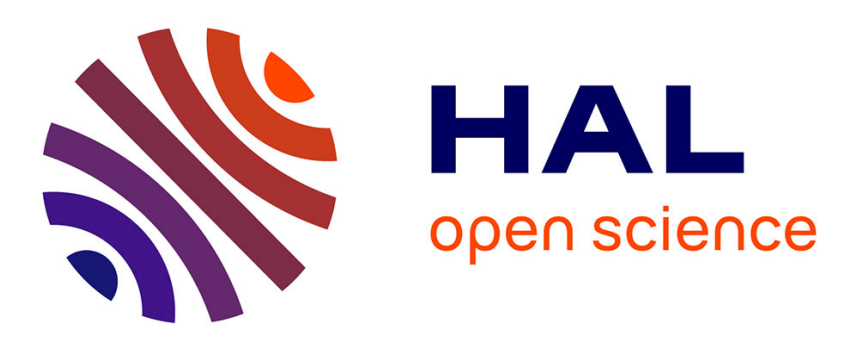

\title{
Hybrid analytical and extended finite element method (HAX-FEM): A new enrichment procedure for cracked solids
}

\author{
Julien Réthoré, Stéphane Roux, François Hild
}

\section{To cite this version:}

Julien Réthoré, Stéphane Roux, François Hild. Hybrid analytical and extended finite element method (HAX-FEM): A new enrichment procedure for cracked solids. International Journal for Numerical Methods in Engineering, 2010, 81, pp.269-285. 10.1002/nme.2691 . hal-00430488

\section{HAL Id: hal-00430488 https://hal.science/hal-00430488}

Submitted on 7 Nov 2009

HAL is a multi-disciplinary open access archive for the deposit and dissemination of scientific research documents, whether they are published or not. The documents may come from teaching and research institutions in France or abroad, or from public or private research centers.
L'archive ouverte pluridisciplinaire HAL, est destinée au dépôt et à la diffusion de documents scientifiques de niveau recherche, publiés ou non, émanant des établissements d'enseignement et de recherche français ou étrangers, des laboratoires publics ou privés. 


\title{
Hybrid analytical and extended finite element method (HAX-FEM): A new enrichment procedure for cracked solids
}

\author{
Julien Réthoré ${ }^{1, * \dagger}$, Stéphane Roux ${ }^{2}$ and François Hild ${ }^{2}$ \\ ${ }^{1}$ Laboratoire de Mécanique des Contacts et des Structures (LaMCoS), INSA Lyon/Université de Lyon/UMR CNRS \\ 5259, 20 Avenue des Sciences, F-69621 Villeurbanne Cedex, France \\ ${ }^{2}$ Laboratoire de Mécanique et Technologie (LMT-Cachan), ENS-Cachan/CNRS UMR 8535/Université Paris \\ VI/PRES UniverSud Paris, 61 Avenue du Président Wilson, F-94235 Cachan Cedex, France
}

\section{SUMMARY}

A new numerical method is proposed to estimate directly stress intensity factors at the tip of a crack in an elastic body. In the same spirit as for the extended finite element method, the approximation of the displacement field is enriched in the vicinity of the crack tip. Yet the method proposed herein differs by the way the enrichment is introduced. Instead of using partition of unity concepts, a two-description formulation is implemented. The first one uses standard finite elements while the second one, in the vicinity of the crack tip, resorts to a few purely analytical expressions. These two descriptions are then coupled by partitioning the energy with an overlapping zone. The performance of this new enrichment technique is illustrated and compared with existing techniques by means of two examples. Copyright (C) 2009 John Wiley \& Sons, Ltd.

Received 9 December 2008; Revised 3 April 2009; Accepted 27 May 2009

KEY WORDS: X-FEM; Williams' series; energy coupling; stress intensity factors

\section{INTRODUCTION}

Since the pioneering papers of Black and Belytschko [1] and Moës et al. [2], the extended finite element method (X-FEM), initially dedicated to elastic solids, was extensively developed to deal with many applications. Concerning the simulation of failure in solids, 3D crack propagation [3, 4], dynamic crack propagation [5-10] were simulated, non-linear models [11,12], and multi-grid solvers [13] were implemented, and coupling with molecular models $[14,15]$ was shown to be

\footnotetext{
*Correspondence to: Julien Réthoré, Laboratoire de Mécanique des Contacts et des Structures (LaMCoS), INSA Lyon, 20 Avenue des Sciences, F-69621 Villeurbanne Cedex, France.

${ }^{\dagger}$ E-mail: Julien.Rethore@insa-lyon.fr

Contract/grant sponsor: CETIM Foundation

Copyright (C) 2009 John Wiley \& Sons, Ltd.
} 
possible. Even full field measurement techniques such as digital image correlation [16-19] now use enriched kinematics.

$\mathrm{X}$-FEM rests on different enrichments, namely, displacement discontinuities across the crack faces, and (standard crack) singular functions at the crack tip. Ironically, the latter enrichments cannot be trusted to provide reliable estimates of stress intensity factors (SIFs) [20]. Hence, most of these analyses require a post-processing stage to extract SIFs from the simulated displacement field. This stage is usually performed by using interaction integrals [1] or least-squares techniques [6].

In References [21,22], an alternative enrichment basis is proposed where SIFs $K_{\mathrm{I}}$ and $K_{\mathrm{II}}$ are directly evaluated as parameters of the discretization basis. It is referred to as KX-FEM. It is shown in these references that nodal enriched degrees freedom corresponding to $K_{\mathrm{I}}$ and $K_{\mathrm{II}}$ must be constrained to have the same value. This strategy is generalized in Reference [23] by the use of a cut-off function. Other requirements to get accurate SIFs are to include in the basis not only the usual asymptotic terms (with $K_{\mathrm{I}}$ and $K_{\mathrm{II}}$ as pre-factors) but also higher-order terms [21].

Having implemented enriched kinematics in a digital image correlation framework [18] spurred the present authors to work on the improvement of the robustness of SIF extraction techniques. For instance, noise robust interaction integrals [24] but also a general formalism of the extraction problem (Réthoré et al., submitted 2008) were recently proposed. In the latter reference, one of the conclusions was that the best determination of SIFs is obtained when they are directly the unknowns of the correlation problem as in integrated approaches of digital image correlation [25]. One of the driving idea of the present paper is to propose a numerical methodology that allows for direct extractions of SIFs with no additional data processing.

Although the method proposed by Liu et al. [21] and Xiao and Karihaloo [22] is very appealing, it will be shown herein that the underlying partition of unity that is kept active over the enriched region reduces drastically the potential of the method. It can be traced back to the conditioning of the system $[20,23,26]$. By reducing the number of degrees of freedom of the enrichment leads to a better conditioning, and hence a more accurate evaluation of SIFs. An alternative to the partition of unity enrichment is proposed herein. An analytical model written in Williams' series formalism [27] is used in the vicinity of the crack tip where the finite element model is not activated. The analytical model includes rigid body translations, rotation, classical asymptotic terms (and $T$-stress and higher-order term can also be included). Then both descriptions are matched using an energy coupling procedure or Arlequin method [28].

Section 2 briefly recalls the family of functions describing the displacement of a cracked elastic solid. In Section 3, the new formulation is proposed and its discretization is discussed in Section 4. In Section 5, the performance of the proposed enrichment technique is illustrated with two examples.

\section{CRACK DISPLACEMENT FIELD}

Let us consider a homogeneous body with isotropic elastic behavior, and a 2D setting, the displacement field $\mathbf{u}$ is conventionally represented by its complex writing, $\mathbf{u}=u_{x}+\mathrm{i} u_{y}$. It was expanded by Williams [27] for a straight crack as a double series

$$
\mathbf{u}(r, \theta)=\sum_{\mathrm{i}=\mathrm{I}, \mathrm{II}} \sum_{n} c_{i}^{n} \boldsymbol{\phi}_{i}^{n}(r, \theta)
$$


where $c_{i}^{n}$ are the influence parameters, and $\phi_{i}^{n}$ reference fields that form the complete set of linear elastic fields satisfying a zero traction condition along the crack path

- even $n$

$$
\begin{aligned}
& \phi_{\mathrm{I}}^{n}(r, \theta)=r^{n / 2}\left(\kappa \mathrm{e}^{\mathrm{i} n \theta / 2}-\frac{n}{2} \mathrm{e}^{i(4-n) \theta / 2}+\left(\frac{n}{2}+1\right) \mathrm{e}^{-\mathrm{i} n \theta / 2}\right) \\
& \phi_{\mathrm{II}}^{n}(r, \theta)=i r^{n / 2}\left(\kappa \mathrm{e}^{\mathrm{i} n \theta / 2}+\frac{n}{2} \mathrm{e}^{i(4-n) \theta / 2}-\left(\frac{n}{2}-1\right) \mathrm{e}^{-\mathrm{i} n \theta / 2}\right)
\end{aligned}
$$

- odd $n$

$$
\begin{aligned}
& \phi_{\mathrm{I}}^{n}(r, \theta)=(-1)^{(n+1) / 2} r^{n / 2}\left(\kappa \mathrm{e}^{\mathrm{i} n \theta / 2}-\frac{n}{2} \mathrm{e}^{i(4-n) \theta / 2}+\left(\frac{n}{2}-1\right) \mathrm{e}^{-\mathrm{i} n \theta / 2}\right) \\
& \phi_{\mathrm{II}}^{n}(r, \theta)=i(-1)^{(n-1) / 2} r^{n / 2}\left(\kappa \mathrm{e}^{\mathrm{i} n \theta / 2}+\frac{n}{2} \mathrm{e}^{\mathrm{i}(4-n) \theta / 2}-\left(\frac{n}{2}+1\right) \mathrm{e}^{-\mathrm{i} n \theta / 2}\right)
\end{aligned}
$$

where $\kappa$ is the Kolossov constant, namely, $\kappa=(3-v) /(1+v)$ for plane stress or $\kappa=(3-4 v)$ for plane strain conditions, $v$ being Poisson's ratio.

The partition between modes I and II is performed on the basis of the following symmetries

$$
\begin{aligned}
\phi_{\mathrm{I}}^{n}(\overline{\mathbf{z}}) & =\overline{\phi_{\mathrm{I}}^{n}(\mathbf{z})} \\
\phi_{\mathrm{II}}^{n}(\overline{\mathbf{z}}) & =-\overline{\boldsymbol{\phi}_{\mathrm{II}}^{n}(\mathbf{z})}
\end{aligned}
$$

All these fields are homogeneous functions of the distance to the crack tip $r$. With this convention, all elastic displacement fields with finite strain energy densities are generated with $0 \leqslant n<\infty$. All odd indices label fields with a discontinuity across the crack mouth, whereas even indices correspond to continuous fields. To mention just a few examples, $n=0$ corresponds to translations either parallel (hence termed I) or perpendicular (labeled II) to the crack path, $n=1$ are the classical mode I and mode II displacement fields whose amplitudes are proportional to the corresponding SIFs. The case $n=2$ gives access to either the $T$-stress component for mode I, or rigid body rotation about the crack tip for mode II (no discontinuity is involved here since 2 is even). Larger indices correspond to 'subsingular' or higher-order fields that may capture the remote heterogeneity of the loading, but do not affect the fracture behavior at the crack tip. This family of fields is thus the appropriate basis function to describe the displacement field for a traction-free crack in an elastic solid.

\section{PROBLEM FORMULATION}

Owing to the difficulties described in the introduction, one wants to enrich a finite element discretization using the family of functions introduced above, but not in the standard extended finite element formalism. The underlying partition of unity must be deactivated over the region where the enriched part of the displacement basis is adopted. For this purpose, the energy coupling (or Arlequin) procedure proposed by Ben Dhia and Rateau [28] is chosen.

For each description of the displacement field, the derived elastic stress must satisfy the balance of momentum (body forces are neglected herein)

$$
\operatorname{div} \boldsymbol{\sigma}=0
$$




\section{J. RÉTHORÉ, S. ROUX AND F. HILD}

where $\boldsymbol{\sigma}$ is Cauchy's stress tensor, and div the divergence operator. The domain $\Omega$ is now subdivided into two overlapping sub-domains $\Omega_{1}$ and $\Omega_{2}$. In each of these sub-domains, a description $\mathbf{u}_{1}$, respectively, $\mathbf{u}_{2}$, of the displacement field is adopted. The boundary conditions are held by the sub-domain $\Omega_{1}$, namely, tractions $\mathbf{t}_{d}$ and displacements $\mathbf{u}_{d}$ are prescribed on two complementary parts $\partial \Omega_{1 t}$ and $\partial \Omega_{1 u}$ of its boundary.

From the strong form of the equilibrium equation (7), two weak forms (one for each description) are derived, and are weighted by a partition of unity over the overlapping region $\Omega_{12}=\Omega_{1} \cap \Omega_{2}$

$$
\alpha_{1}(\mathbf{x})+\alpha_{2}(\mathbf{x})=1
$$

where $\alpha_{1}$ and $\alpha_{2}$ are the respective weights given to each energy contribution. The definition of these weighting functions is extended over the entire domain $\Omega$ so that the description 1 (resp. 2) holds in $\Omega \backslash \Omega_{2}$ only (resp. $\Omega \backslash \Omega_{1}$ ). In this setting, the linear $l_{i}$ and bilinear $a_{i}$ weighted energy forms are obtained for $i=1$ or 2

$$
\begin{aligned}
a_{i}\left(\mathbf{u}_{i}, \mathbf{v}_{i}^{*}\right) & =\int_{\Omega_{i}} \alpha_{i} \boldsymbol{\varepsilon}\left(\mathbf{u}_{i}\right): \mathbb{C}: \boldsymbol{\varepsilon}\left(\mathbf{v}_{i}^{*}\right) \mathrm{d} \Omega \\
l_{1}\left(\mathbf{v}_{1}^{*}\right) & =\int_{\partial \Omega_{1 t}} \alpha_{1} \mathbf{t}_{d} \cdot \mathbf{v}_{1}^{*} \mathrm{~d} S
\end{aligned}
$$

where $\mathbb{C}$ is Hooke's tensor, $\boldsymbol{\varepsilon}$ the infinitesimal strain tensor, and $\mathbf{v}_{i}^{*}$ admissible displacement fields. Note that $l_{2}$ is 0 since the crack faces are traction free.

In addition to this partition of energy, a kinematic coupling must be added to ensure the compatibility of the two displacement fields in the sense of a scalar product $\Pi$ between the displacement gap and Lagrange multipliers $\lambda$. If $\Pi$ is the $L_{2}$ scalar product over the coupling region $\Omega_{12}$, we have

$$
\Pi\left(\mathbf{u}_{1}-\mathbf{u}_{2}, \boldsymbol{\mu}^{*}\right)=\int_{\Omega_{12}} \boldsymbol{\mu}^{*} \cdot\left(\mathbf{u}_{1}-\mathbf{u}_{2}\right) \mathrm{d} \Omega=0
$$

where $\boldsymbol{\mu}^{*}$ are admissible Lagrange multipliers. The total formulation thus reads: find $\left(\mathbf{u}_{1}, \mathbf{u}_{2}, \boldsymbol{\lambda}\right)$ such that for all admissible $\left(\mathbf{v}_{1}^{*}, \mathbf{v}_{2}^{*}, \boldsymbol{\mu}^{*}\right)$ fields,

$$
a_{1}\left(\mathbf{u}_{1}, \mathbf{v}_{1}^{*}\right)+a_{2}\left(\mathbf{u}_{2}, \mathbf{v}_{2}^{*}\right)+\Pi\left(\mathbf{u}_{1}-\mathbf{u}_{2}, \boldsymbol{\mu}^{*}\right)+\Pi\left(\mathbf{v}_{1}^{*}-\mathbf{v}_{2}^{*}, \lambda\right)=l_{1}\left(\mathbf{v}_{1}^{*}\right)+l_{2}\left(\mathbf{v}_{2}^{*}\right)
$$

Using this weak formulation, a discretization scheme is being adopted for the two descriptions.

\section{DISCRETIZATION AND NUMERICAL ELABORATION}

After the weak formulation is chosen, the discretization schemes for the two descriptions and some details on the numerical elaboration are detailed in the sequel.

\subsection{Outer domain (Model 1)}

For the outer domain, model 1 , a finite element discretization is adopted. $\Omega$ is divided into a given number of elements, a subset being 'active' as they define the discretized domain $\Omega_{1}^{h}$ and the complementary subset being 'inactive'. Further, a discontinuous enrichment is added to the 
interpolation to account for the displacement jump across the part of the crack faces that lies in $\Omega_{1}$. The interpolation of the displacement for the outer model thus reads

$$
\mathbf{u}_{1}(\mathbf{x})=\sum_{i \in \mathscr{N}_{1}} \mathbf{N}_{i}(\mathbf{x}) d_{i}+\sum_{i \in \mathscr{N}_{\text {cut }}} \mathbf{N}_{i}(\mathbf{x}) \mathscr{H}(\mathbf{x}) b_{i}=\mathbf{D}^{\mathrm{T}} \mathbf{U}_{1}
$$

where $\mathbf{N}_{i}$ are standard finite element shape functions supported by the set of nodes $\mathscr{N}_{1}$ included in $\Omega_{1}^{h}, d_{i}$ the corresponding degrees of freedom. In this equation, $\mathscr{H}(\mathbf{x})$ is the discontinuous symmetrized Heaviside step function that enables with its associated degrees of freedom $b_{i}$ for the description of displacement discontinuities for the nodes in the subset $\mathscr{N}_{\text {cut }}$ of $\mathscr{N}_{1}$ that have their support cut by the crack. This interpolation relies on a usual extended finite element discretization based on the partition of unity property of $\mathbf{N}_{i}$ [29]. Matrix $\mathbf{D}$ collects the value of the discretization functions and vector $\mathbf{U}_{1}$ the degrees of freedom of the outer domain.

In the following, only regular meshes of quadrangular elements will be used. For the numerical integration of the discontinuous function in the weak form, the quadrangles are subdivided into $n_{\text {sub }} \times n_{\text {sub }}$ quadrangular integration cells containing one Gauss point. Standard Gauss quadrature rule with four integration points per quadrangle is used for the standard terms.

\subsection{Inner domain (Model 2)}

For the inner domain, model 2, the functions are selected in the basis presented in Section 2. The displacement field $\mathbf{u}_{2}(\mathbf{x})$ reads

$$
\mathbf{u}_{2}(\mathbf{x})=\frac{1}{2 \mu \sqrt{2 \pi}} \sum_{n \in\left[0 ; n_{\max }\right]}\left(\phi_{\mathrm{I}}^{n}(\mathbf{x}) p_{n}+\boldsymbol{\phi}_{\mathrm{II}}^{n}(\mathbf{x}) q_{n}\right)=\boldsymbol{\Phi}^{\mathrm{T}} \mathbf{U}_{2}
$$

where $\mu$ is Lamé's coefficient, $p_{n}$ and $q_{n}$ the degrees of freedom associated with mode I and II functions, and $n_{\max }$ an integer that defines the maximum order considered in the interpolation. Matrix $\boldsymbol{\Phi}$ collects the values of the discretization functions and vector $\mathbf{U}_{2}$ the degrees of freedom of the inner domain. Note that for $n=1, p_{1}$ and $q_{1}$ enable for a direct evaluation of $K_{\mathrm{I}}$ and $K_{\mathrm{II}}$. The discretized domain $\Omega_{2}^{h}$ is defined as the area of the 'inactive' finite elements plus those in the coupling zone $\Omega_{12}^{h}$. The numerical integration of the weak form $a_{2}$ is performed in the same way for the enriched terms of $a_{1}$, namely, by using the underlying finite elements that are subdivided into $n_{\text {sub }} \times n_{\text {sub }}$ quadrangular integration cells containing one Gauss point. The discretization functions adopted for the inner domain satisfy Equation (7) and thus the integration of $a_{2}$ could be split into two domain integrals (the first one defined on the domain for which $\alpha_{2}=1$ vanishes, and the second one in $\Omega_{12}^{h}$ ), and a contour integral of the normal tractions on the inner boundary of

$\Omega_{12}^{h}$. Up to the influence of the weighting function, this contour integral is exactly the one that is required for the hybrid crack element (see e.g. [30]). In practice, it is more convenient to integrate the initial form of $a_{2}$.

\subsection{Coupling}

The discretized coupling domain is the subset of elements in $\Omega_{12}^{h}$. Lagrange multipliers are discretized using standard finite element shape functions

$$
\lambda(\mathbf{x})=\sum_{i \in \mathscr{N}_{12}} \mathbf{N}_{i}(\mathbf{x}) l_{i}=\mathbf{L}^{\mathrm{T}} \boldsymbol{\Lambda}
$$




\section{J. RÉTHORÉ, S. ROUX AND F. HILD}

where $\mathscr{N}_{12}$ is the set of nodes in $\Omega_{12}^{h}, \mathbf{N}_{i}$ standard finite element shape functions, and $l_{i}$ their associated degrees of freedom. Matrix $\mathbf{L}$ collects the values of the discretization functions and vector $\Lambda$ the degrees of freedom of Lagrange multipliers. Since the coupling terms involve discontinuous functions and basis functions of description 2, the above-mentioned quadrature using cells with one Gauss point is used.

\subsection{Numerical elaboration}

After the discretization schemes have been introduced in the weak form, a linear system is obtained

$$
\left[\begin{array}{ccc}
\mathbf{K}_{1} & \mathbf{0} & \mathbf{C}_{1}^{\mathrm{T}} \\
\mathbf{0} & \mathbf{K}_{2} & \mathbf{C}_{2}^{\mathrm{T}} \\
\mathbf{C}_{1} & \mathbf{C}_{2} & \mathbf{0}
\end{array}\right]\left[\begin{array}{c}
\mathbf{U}_{1} \\
\mathbf{U}_{2} \\
\boldsymbol{\Lambda}
\end{array}\right]=\left[\begin{array}{c}
\mathbf{F}_{1} \\
\mathbf{F}_{2} \\
\mathbf{0}
\end{array}\right]
$$

with

$$
\begin{aligned}
& \mathbf{K}_{1 i j}=\int_{\Omega_{1}^{h}} \alpha_{1} \boldsymbol{\varepsilon}\left(\mathbf{D}_{i}\right): \mathbb{C}: \boldsymbol{\varepsilon}\left(\mathbf{D}_{j}\right) \mathrm{d} \Omega \\
& \mathbf{K}_{2 i j}=\int_{\Omega_{2}^{h}} \alpha_{2} \boldsymbol{\varepsilon}\left(\boldsymbol{\Phi}_{i}\right): \mathbb{C}: \boldsymbol{\varepsilon}\left(\boldsymbol{\Phi}_{j}\right) \mathrm{d} \Omega \\
& \mathbf{C}_{1 i j}=\int_{\Omega_{12}^{h}} \mathbf{L}_{i} \cdot \mathbf{D}_{j} \mathrm{~d} \Omega \\
& \mathbf{C}_{2 i j}=-\int_{\Omega_{12}^{h}} \mathbf{L}_{i} \cdot \boldsymbol{\Phi}_{j} \mathrm{~d} \Omega
\end{aligned}
$$

are elementary blocks of the global matrix and

$$
\begin{aligned}
\mathbf{F}_{1 i} & =\int_{\partial \Omega_{t}} \alpha_{1} \mathbf{t}_{d} \cdot \mathbf{D}_{i} \mathrm{~d} S \\
\mathbf{F}_{2 i} & =\int_{\partial \Omega_{t}} \alpha_{2} \mathbf{t}_{d} \cdot \boldsymbol{\Phi}_{i} \mathrm{~d} S
\end{aligned}
$$

the external force vectors. Note that, in practice, $\mathbf{F}_{2}$ is equal to zero.

For practical reasons, the weighting functions $\alpha_{i}$ are computed numerically by solving a Laplacian problem $\left(\Delta \alpha_{i}=0\right)$ using a finite element method in the coupling zone $\Omega_{12}^{h}$. For example,

$\alpha_{1}$ is computed using the appropriate boundary conditions on the boundary of $\Omega_{12}^{h}$ and $\alpha_{2}$ is then directly obtained using Equation (8).

\section{EXAMPLES}

In the following, two examples are discussed. The first one corresponds to a closed-form solution of a crack in an infinite domain, i.e. the extension of the inner field to infinity, and it will test the 
compatibility of the entire global scheme. The second one is more informative as it corresponds to a problem that has no exact (closed-form) solution.

\subsection{Infinite plate with a semi-infinite crack}

The first example is dedicated to a convergence study in the idealized case of an infinite plate with a semi-infinite crack. The numerical model consists of a $39 \times 39$ quadrangular elements with a horizontal centered crack whose tip is located in the middle of the plate. To fall within the hypothesis of an infinite plate, asymptotic fields for mode I, with $K_{\mathrm{I} o}$ as a prescribed SIF, and mode II, with $K_{\text {II } o}$ as a prescribed SIF, are imposed on the outer boundary of the mesh. The plate is considered to be of area $1 \mathrm{~m}^{2}$, the material properties are set to $210 \mathrm{MPa}$ for Young's modulus and 0.3 for Poisson's ratio. The prescribed SIF values are $1 \mathrm{MPa} \sqrt{\mathrm{m}}$ for $K_{\mathrm{I} o}$ and $K_{\mathrm{II} o} .16 \times 16$ quadrature cells in each quadrangles are used where needed. Let us focus on the influence of the size of the analytical model (that contains indices ranging between 0 and 2, i.e. $n_{\max }=2$ ) and also on the size of the coupling zone.

The size of $\Omega_{2}^{h}$ is defined as $R_{\text {outer }}=h r_{\text {outer }}$ ( $h$ being the element size), and that of 'inactive' elements $R_{\text {inner }}=r_{\text {inner }} h$. The size of the coupling region is $h \ell_{\text {overlap }}=R_{\text {outer }}-R_{\text {inner }}$. As an example, Figure 1 shows the weighting function $\alpha_{1}$ of the finite element model for an analytical model of 10-node layer around the crack tip, $r_{\text {inner }}=10$ and an overlapping zone of 5-node layer, $\ell_{\text {overlap }}=5$. Figure 2 shows the two components of the displacement field for the two models when $r_{\text {inner }}=3$ and $\ell_{\text {overlap }}=3$.

In order to study the influence of the size of the inner domain and its coupling with the FE zone (outer domain), $r_{\text {inner }}$ is varied in the interval $1 \leqslant r_{\text {inner }} \leqslant 10$ and $1 \leqslant \ell_{\text {overlap }} \leqslant 5$. Figures 3 and 4 summarize the results. In Figure 3, the relative errors on $K_{\mathrm{I}}$ and $K_{\mathrm{II}}$ are plotted as functions of $r_{\text {inner }}$ for different coupling zone sizes $\ell_{\text {overlap }}$. From the plots using a linear scale, a good convergence is observed. It is to be noted that estimated $K_{\mathrm{I}} \mathrm{s}$ are first less than the prescribed value, then higher and finally converge to $K_{\mathrm{I} o}$ itself. For mode II, only higher values are observed. The log-scale plots show the same trends for mode I and mode II, namely, a linear decrease of the absolute

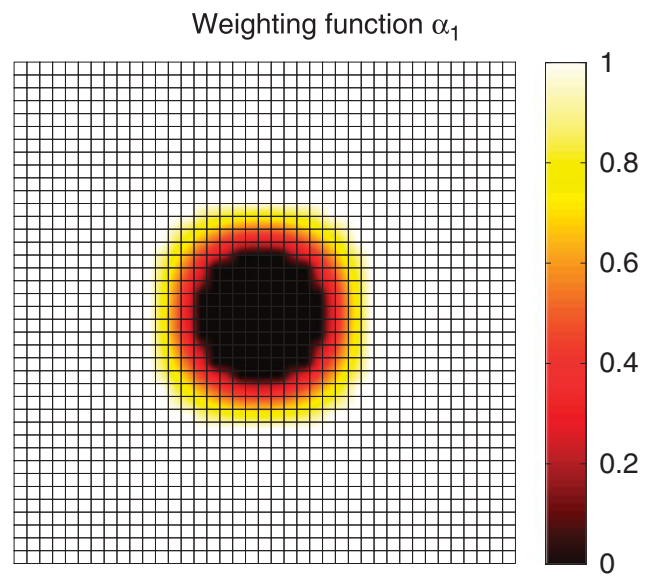

Figure 1. Weighting function of the finite element model $\alpha_{1}$ for the square plate with an edge crack. 

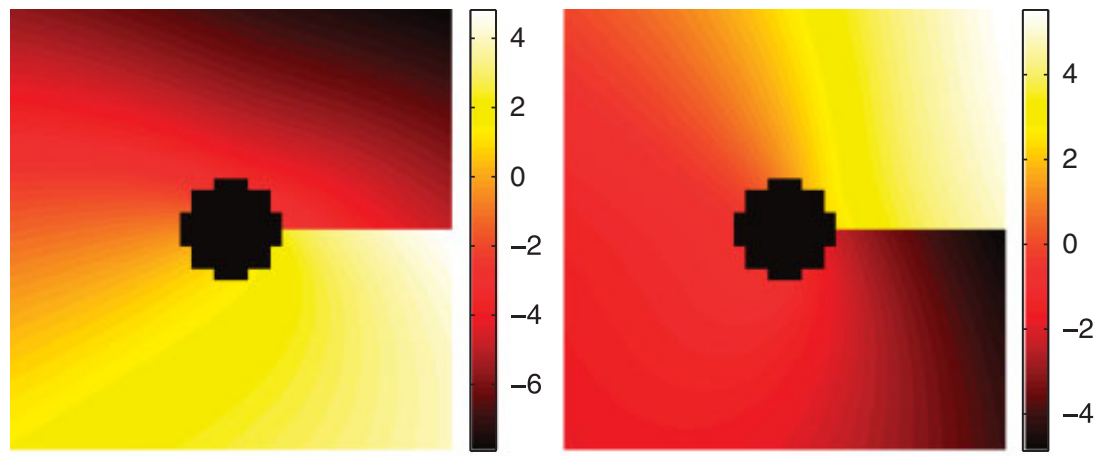

(a)

(b)
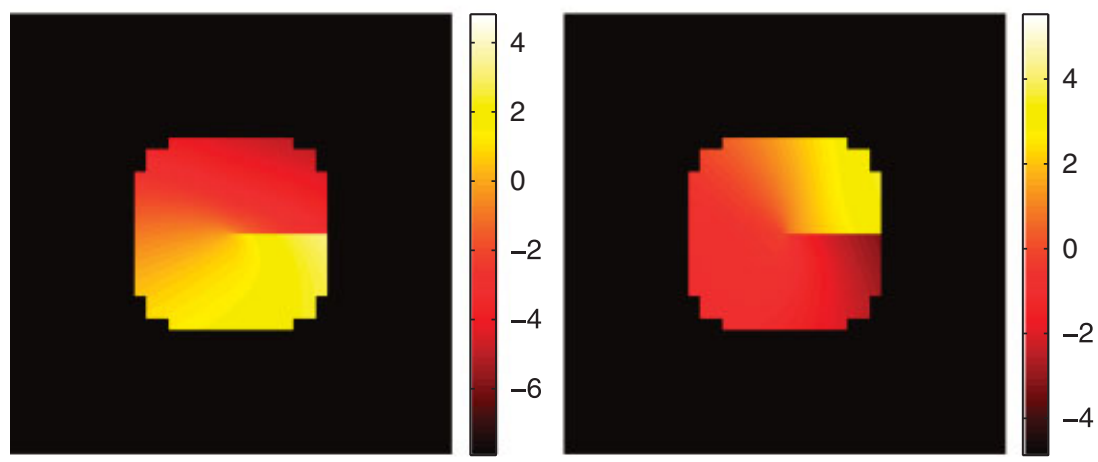

(c)

(d)
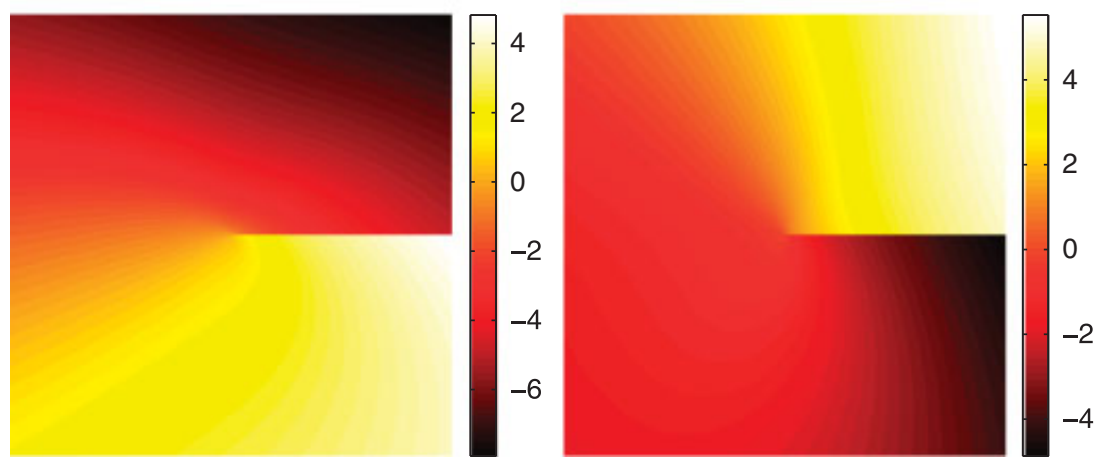

(e)

(f)

Figure 2. Horizontal (left) and vertical (right) displacement field (in $\mu \mathrm{m}$ ) for the square plate. The size of the analytical domain is $r_{\text {inner }}=3$ and the width of the coupling region is $\ell_{\text {overlap }}=3$. The top figures show the displacement in the finite element model, the middle figures in the analytical model and the bottom figures the total displacement.

relative error is obtained, the size of the coupling zone acting as a multiplicative pre-factor. For large domain sizes $r_{\text {inner }}=10$, (i.e. $\frac{1}{4}$ of the size of the mesh), absolute errors on $K_{\mathrm{I}}$ as low as $10^{-4}$ and $2 \times 10^{-5}$ on $K_{\text {II }}$ are obtained for $\ell_{\text {overlap }}=5$ coupling zones. 

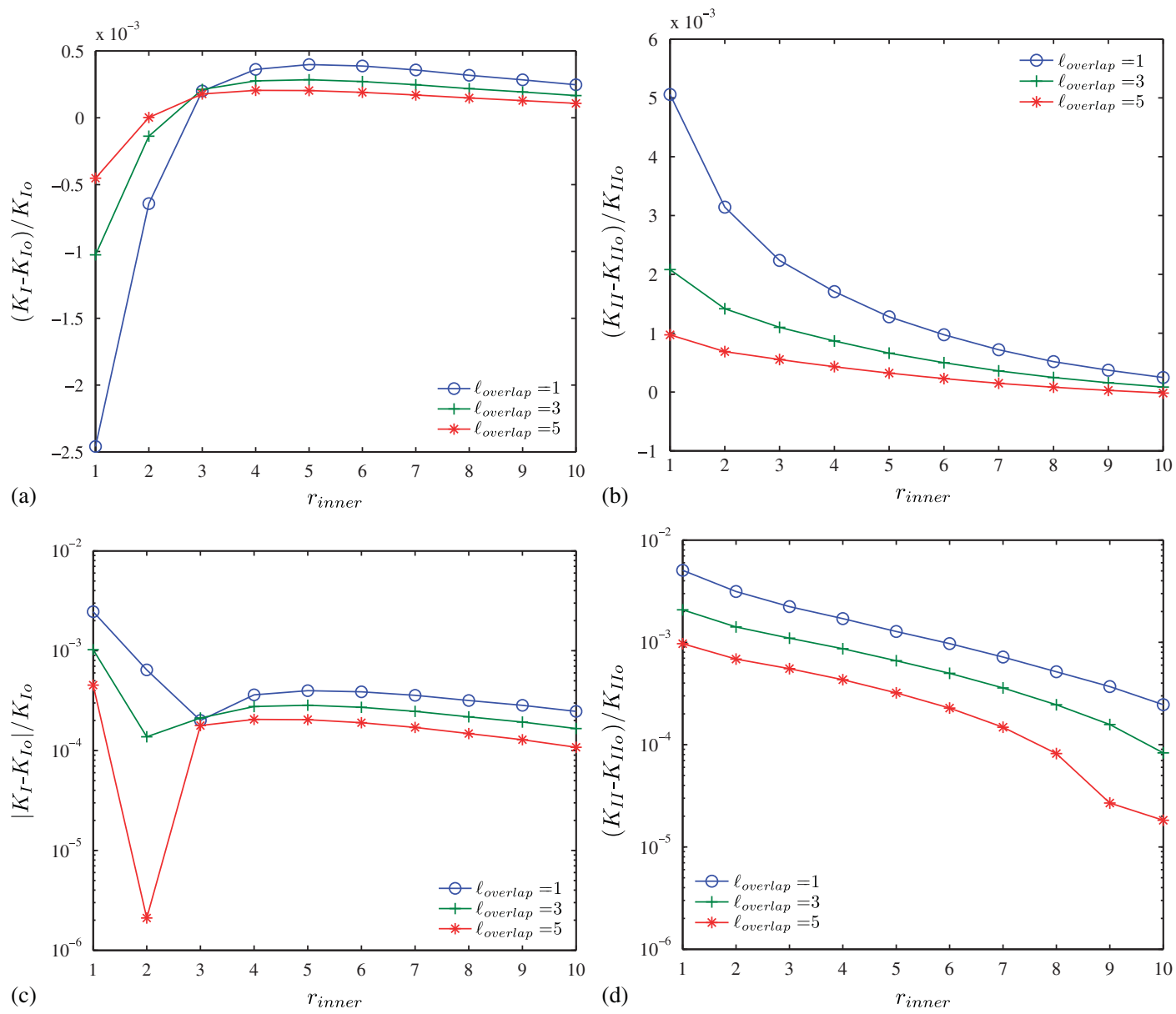

Figure 3. Normalized error on $K_{\mathrm{I}}$ (left) and $K_{\mathrm{II}}$ (right) as functions of the size of the analytical domain $r_{\text {inner. }}$ Top figures are plotted using a linear scale for the $y$-axis whereas the bottom figures use a log scale.

The effect of the size of the coupling zone is shown in Figure 4. The relative error on SIFs follows the same type of dependence with $\ell_{\text {overlap }}$. Even though increasing the size of the analytical model and using larger coupling zone reduces the error on estimated SIFs, the values obtained with as small parameter values $r_{\text {inner }}=\ell_{\text {overlap }}=1$, yields $2.5 \times 10^{-3}$ errors for $K_{\mathrm{I}}$ and $5.0 \times 10^{-3}$ for $K_{\mathrm{II}}$, which are very low values.

\subsection{Cracked plate in remote tension}

In the previous example, because of the idealized boundary conditions, only classical asymptotic terms were activated. This is not the case when realistic boundary conditions are used. The present example aims at evaluating this influence. A cracked plate whose geometry is parameterized by its width $w$, its length $L$ and the length of the crack $a$ is considered. Under remote tension, the 


\section{J. RÉTHORÉ, S. ROUX AND F. HILD}
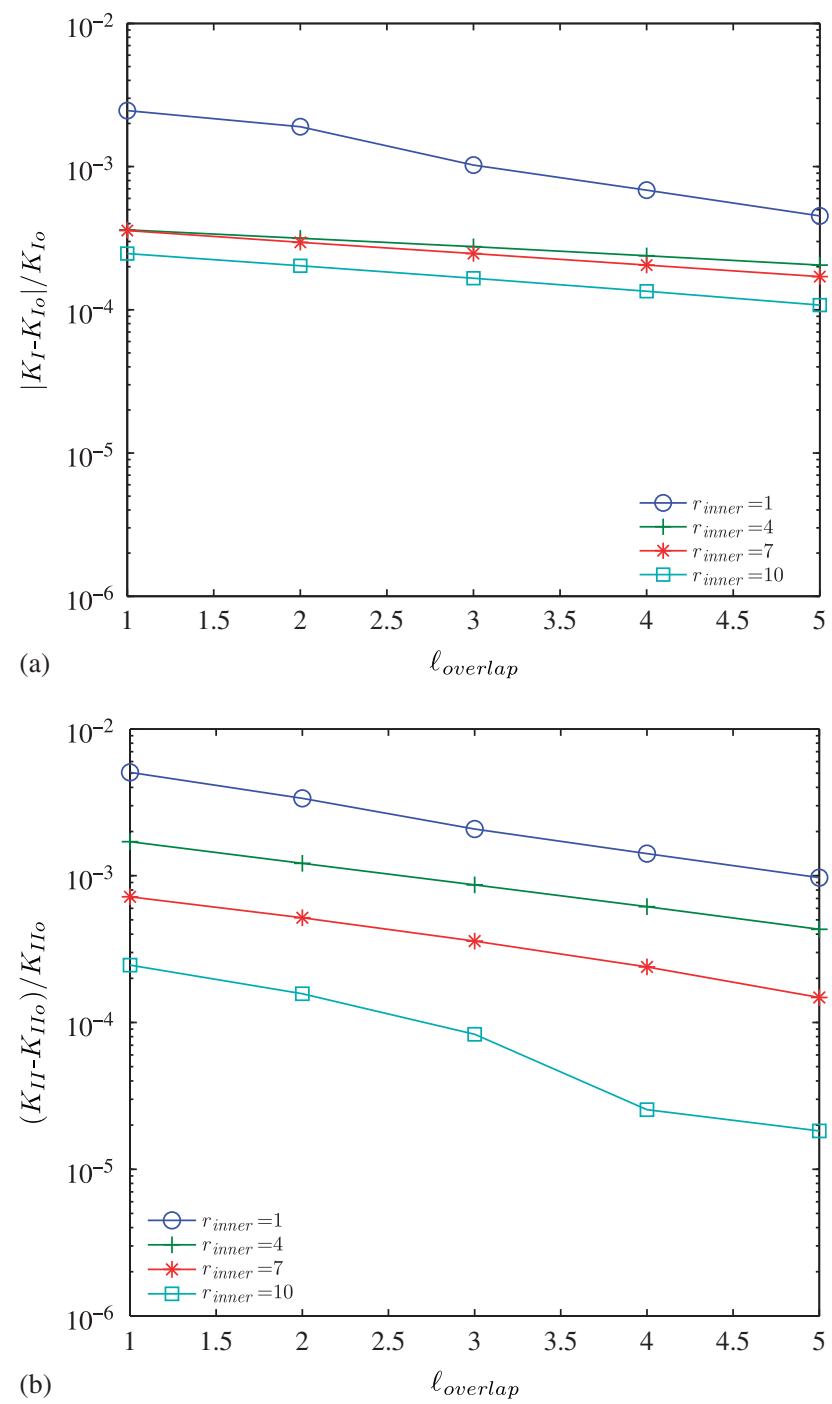

Figure 4. Normalized error on $K_{\mathrm{I}}$ (a) and $K_{\mathrm{II}}$ (b) as functions of width of the coupling zone $\ell_{\text {overlap }}$.

value of $K_{\mathrm{I}}$ that holds for an infinite medium $(\sigma \sqrt{\pi a})$ is corrected by a pre-factor $C$ depending on the ratio $a / w$ only [31]

$$
C=1.12-0.231\left(\frac{a}{w}\right)+10.55\left(\frac{a}{w}\right)^{2}-21.72\left(\frac{a}{w}\right)^{3}+30.39\left(\frac{a}{w}\right)^{4}
$$

In the numerical model $w$ is set to $7 \times 10^{-3} \mathrm{~m}, a$ is equal to $0.5 w$, and $L$ is equal to $17 \times 10^{-3} \mathrm{~m}$. Young's modulus is equal to $200 \mathrm{MPa}$, and Poisson's ratio to 0.3 . The applied stress $\sigma$ is $10 \mathrm{MPa}$, 


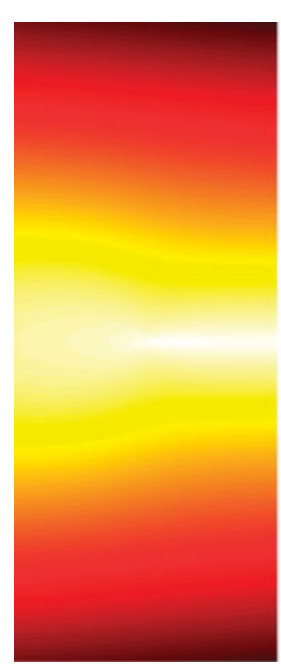

(a)

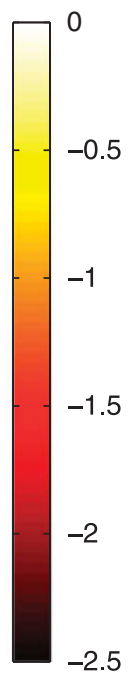

(b)

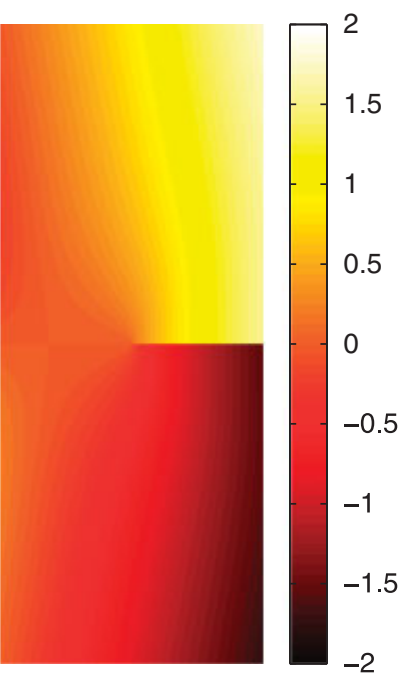

Figure 5. Horizontal (a) and vertical (b) displacement field (in $\mu \mathrm{m}$ ) for the cracked plate in remote tension for $r_{\text {inner }}=\ell_{\text {overlap }}=1$.

which gives $2.98 \mathrm{MPa} \sqrt{\mathrm{m}}$ for $K_{\mathrm{I} o}$ the reference value for $K_{\mathrm{I}}$. The finite element mesh consists of $49 \times 119$ quadrangular elements that are subdivided into $8 \times 8$ integration cells when needed. Figure 5 shows the displacement field for $r_{\text {inner }}=\ell_{\text {overlap }}=1$.

Owing the use of realistic boundary conditions, the normalized values of $K_{\mathrm{I}}$ obtained for $n_{\max }=2$ shown in Figure 6 lead to an error increase when the domain of influence of the analytical model is enlarged (by increasing its size or the size of the coupling zone). The traction-free edge in front of the crack tip activates higher-order term in Williams' series. However, using the smallest domain of influence (i.e. $r_{\text {inner }}=\ell_{\text {overlap }}=1$ ), the normalized value of $K_{\mathrm{I}}$ is 1.0146 (i.e. less than $1.5 \%$ error, which is very low).

In Figure $7, r_{\text {inner }}=10, \ell_{\text {overlap }}=3$ and the effect of the number of terms $n_{\max }$ is investigated. A stabilization of the normalized SIF is observed for a value equal to 1.002, when seven terms are considered. For different values of $n_{\max }$, the normalized value of $K_{\mathrm{I}}$ is plotted as a function of $r_{\text {inner }}$ in Figure 8(a). One notes that the strong dependence observed for $n_{\max }=2$ is decreasing when more terms are considered. For $n_{\max }=7$, the normalized SIF $K_{\mathrm{I}}$ remains almost constant, varying between 0.999 and 1.0012. Let us note that for the smallest domain of influence, $r_{\text {inner }}=\ell_{\text {overlap }}=1$, the normalized value of $K_{\mathrm{I}}$ is 0.999 (i.e. less than $0.1 \%$ error, which is again very low). Figure 8(b) shows the results obtained using KX-FEM under the same conditions. The cut-off function is exactly the weighting function $\alpha_{2}$ and the same number $n_{\max }$ of higher-order terms are considered (only odd terms are introduced in KX-FEM because the underlying partition of unity is assumed to capture the even terms that are not discontinuous across the crack faces). The numerical integration is also performed using the same quadrature. For KX-FEM, an optimal value exists for $r_{\text {inner }} \approx 4-5$ for a normalized value of $K_{1}$ of 0.93 for $n_{\max }=1$ and 0.983 for $n_{\max }=5$. Increasing $n_{\max }$, the results are improved but a critical dependence on $r_{\text {inner }}$ is still observed, namely, for too small $r_{\text {inner }}$, the domain of influence of the enrichment function is not large enough for a robust SIF 


\section{J. RÉTHORÉ, S. ROUX AND F. HILD}

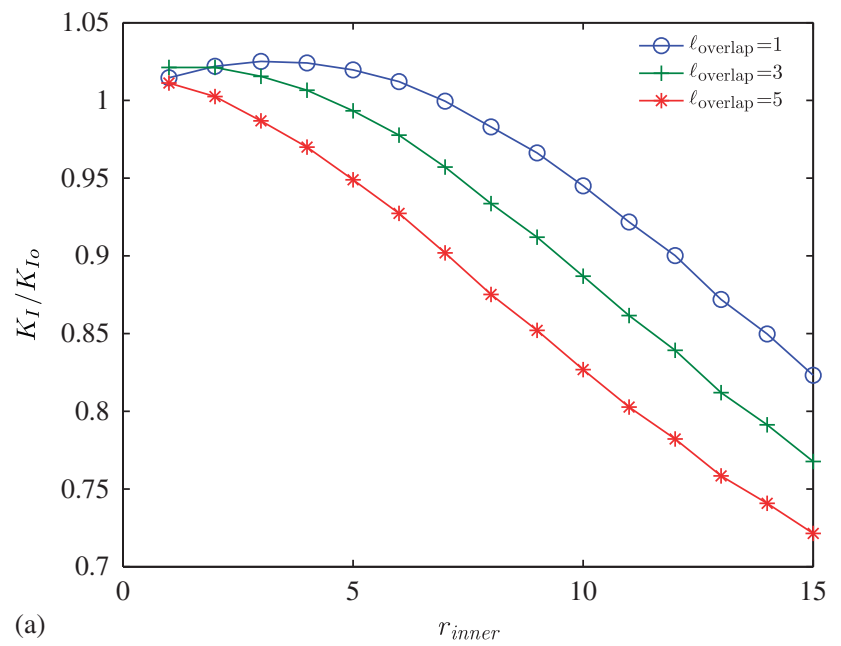

(a)

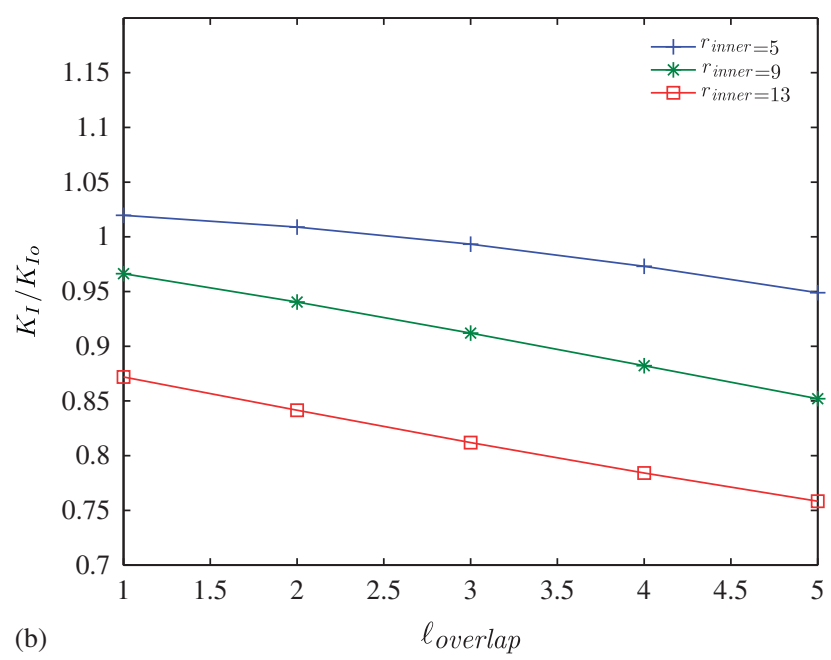

Figure 6. Normalized $K_{\mathrm{I}}$ as function of the size of the analytical domain $r_{\text {inner }}$ (a), the size of the overlapping domain $\ell_{\text {overlap }}$ (b) for $n_{\max }=2$.

estimation but for too large $r_{\text {inner }}$ the conditioning of the system is increasing [20], which also reduces the robustness of the SIF estimation.

For different values of $n_{\max }$, Figure 9(a) shows the effect of the mesh size $h$ while the ratio of the number of elements along the length and width of the sample is kept constant and $r_{\text {inner }}=$ $\ell_{\text {overlap }}=1$. For all values of $n_{\max }$, a standard convergence is obtained with a rate between 1.5 and 1.25 that decreases when $n_{\max }$ increases. Note, that the convergence rate obtained with the present enrichment procedure is faster than that of KX-FEM (Figure 9(b)). Even in its optimal version [20], the latter gives a convergence rate of about 0.5. Here, for comparison purposes, 
HAX-FEM: A NEW ENRICHMENT PROCEDURE FOR CRACKED SOLIDS

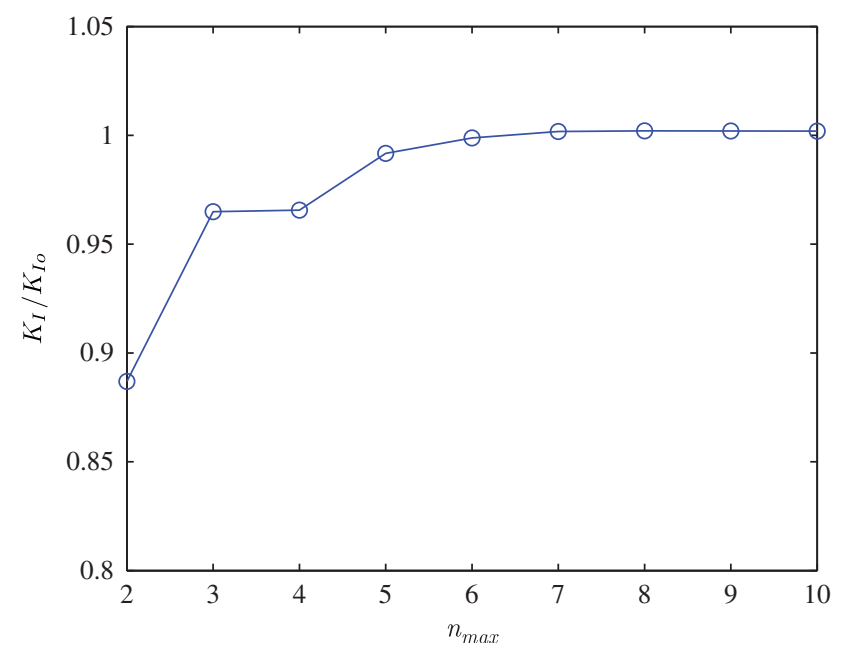

Figure 7. Normalized $K_{\mathrm{I}}$ as function of $n_{\max }$ for $r_{\text {inner }}=10$ and $\ell_{\text {overlap }}=3$.

the cut-off function is equal to $\alpha_{2}$ and thus the outer radius of the domain of influence of the enrichment decreases when the element size decreases $\left(r_{\text {inner }}=\ell_{\text {overlap }}=1\right)$. Further $\alpha_{2}$ is numerically computed using first-order finite elements. It is thus continuous but with discontinuous derivatives, this is not the optimal choice for KX-FEM (Nicaise et al. [20] use an analytically defined fifth-order polynomial cut-off function). However, this crude form of $\alpha_{2}$ seems to be appropriate for the technique proposed herein. We also performed the same convergence study for $\mathrm{KX}$-FEM using $r_{\text {inner }}=4$ and $\ell_{\text {overlap }}=1$. The convergence is improved but no rate faster than 1 was obtained.

This example shows that for realistic boundary conditions, higher-order terms must be considered to obtain low errors on estimated SIF values. As in the previous example, using the proposed technique with the smallest analytical domain and the smallest coupling zone yields already very low errors. This is all the more important if one wants to deal with noisy data when experimental analyses are preformed (e.g. by resorting to DIC (Réthoré et al., submitted 2009)).

\section{CONCLUSION}

The present paper proposes an alternative to partition of unity methods to incorporate enriched interpolation functions. Cracked solids are modeled by using two descriptions that are coupled over a non-zero area region using a partition of energy, namely, using a bridging domain or Arlequin method. The first description consists of standard finite elements in the outer domain, and the second one relies on Williams' series solution in the inner domain. The numerical implementation is described and two examples are proposed to assess the validity of the proposed approach, and to evaluate its performance when seeking stress intensity factors in comparison with usual X-FEM approaches. High accuracy and robustness with respect to the enriched domain size and the overlap width are obtained. Very small inner domains can be used and thus crack 


\section{J. RÉTHORÉ, S. ROUX AND F. HILD}
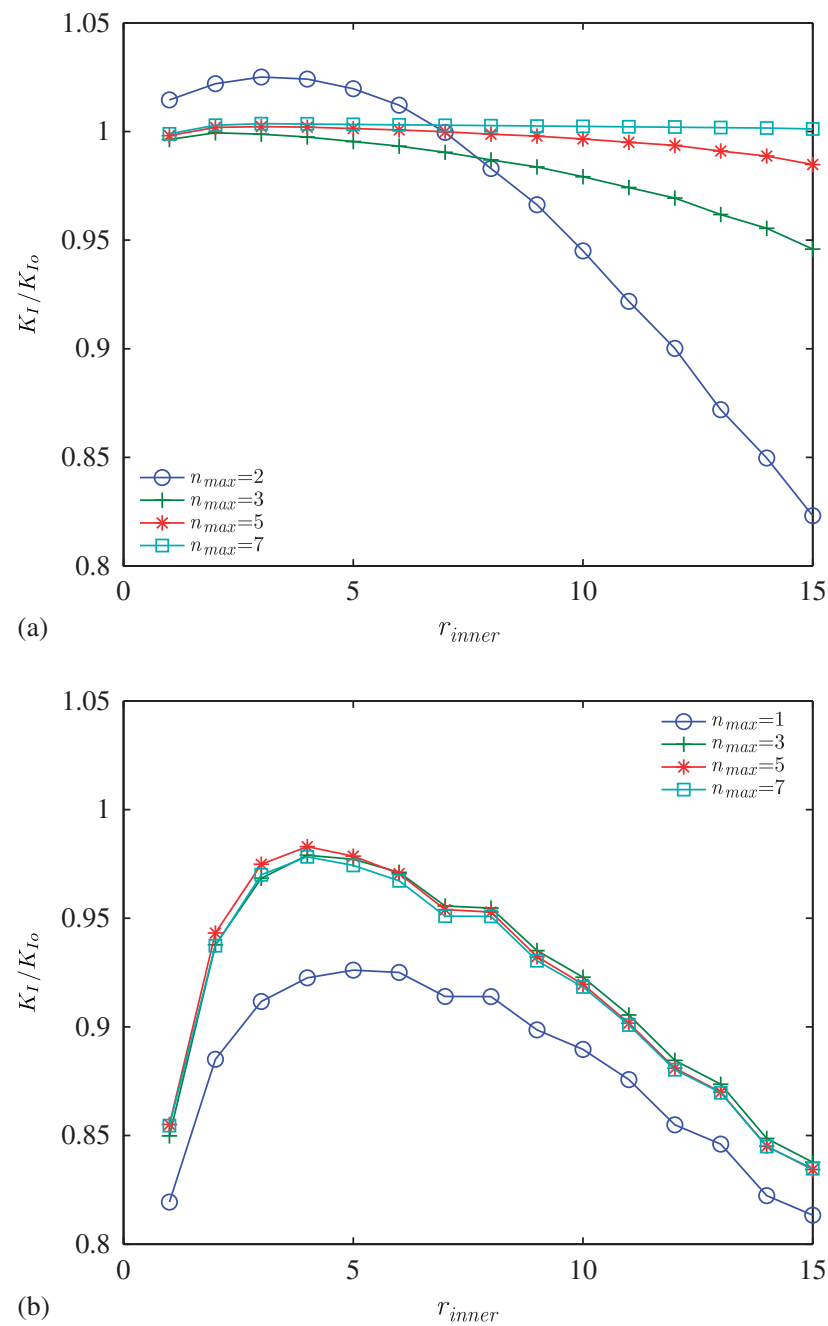

Figure 8. Normalized $K_{\mathrm{I}}$ as function of the size of the analytical domain for different values of $n_{\max }$ obtained using the proposed HAX-FEM (a) and KX-FEM (b).

propagation may also be investigated without any modification of the enriched basis due to the crack curvature.

As a possible extension of the proposed method, analytical crack tip fields with a cohesive law such as those derived by Karihaloo and Xiao [32] may be introduced using the proposed formalism to simulate the cohesive crack growth. Last, the developments discussed herein from a numerical standpoint are currently implemented in an experimental technique to measure displacement fields of crack bodies (by digital image correlation). In that context, the robustness of SIFs extraction is critical since pictures are noisy and the displacement field measurement itself is an ill-posed 
HAX-FEM: A NEW ENRICHMENT PROCEDURE FOR CRACKED SOLIDS
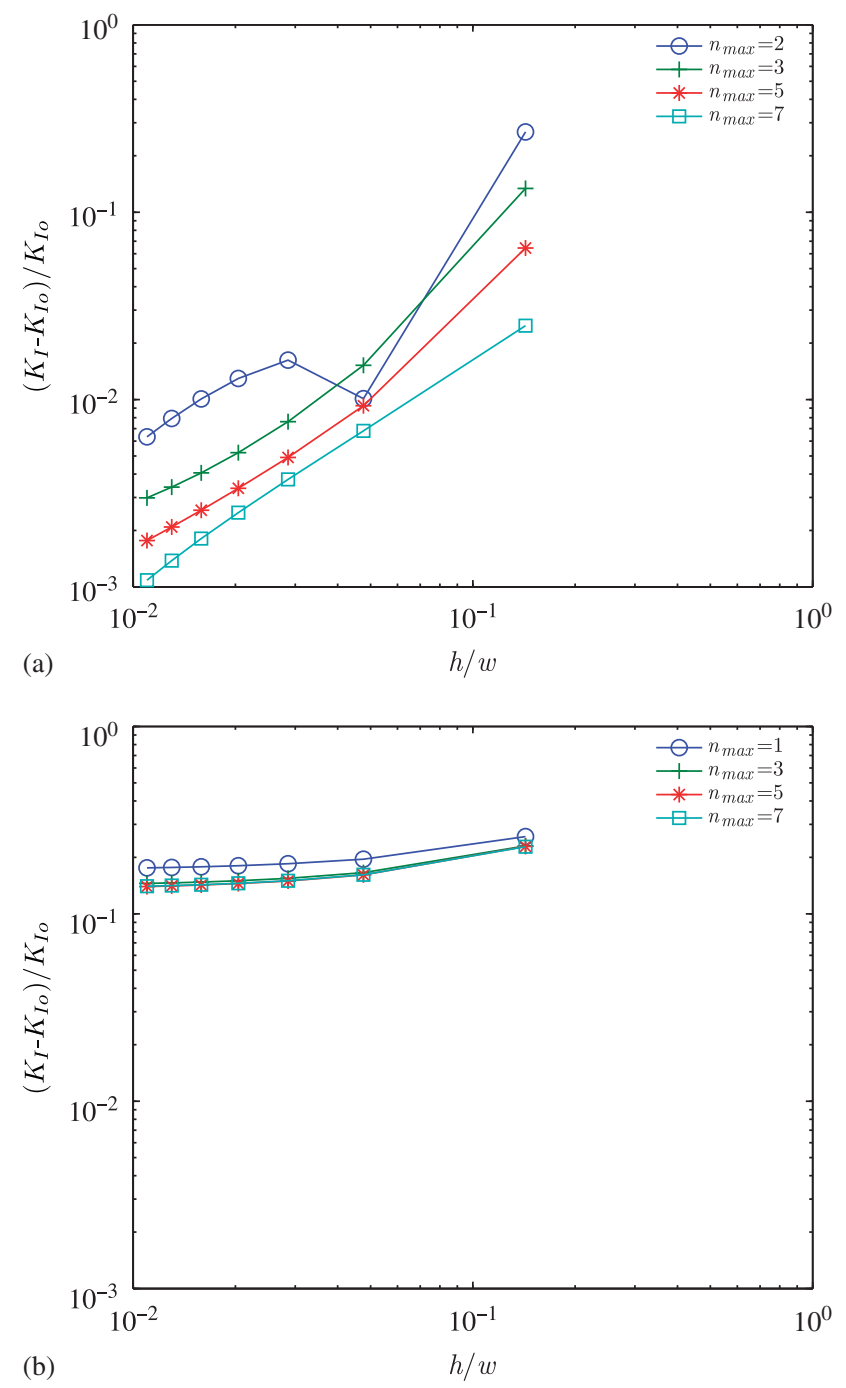

Figure 9. Normalized relative error on $K_{\mathrm{I}}$ as function of the mesh size for different values of $n_{\max }$ obtained using the proposed HAX-FEM (a) and KX-FEM (b) with $r_{\text {inner }}=\ell_{\text {overlap }}=1$.

problem. The fact that the two kinematic bases (numerical and experimental) are identical allows one to avoid interpolation errors and further measurement uncertainties.

\section{ACKNOWLEDGEMENTS}

This work was funded by the CETIM Foundation grant entitled PROPAVANFIS: 'Advanced methods for the experimental and numerical analyses of crack propagations under complex loadings'. 


\section{J. RÉTHORÉ, S. ROUX AND F. HILD}

\section{REFERENCES}

1. Black T, Belytschko T. Elastic crack growth in finite elements with minimal remeshing. International Journal for Numerical Methods in Engineering 1999; 45:601-620.

2. Moës N, Dolbow J, Belytschko T. A finite element method for crack growth without remeshing. International Journal for Numerical Methods in Engineering 1999; 46(1):133-150.

3. Moës N, Gravouil A, Belytschko T. Non-planar 3D crack growth by the extended finite element and level sets. Part I: mechanical model. International Journal for Numerical Methods in Engineering 2002; 53(11):2549-2568.

4. Gravouil A, Moës N, Belytschko T. Non-planar 3D crack growth by the extended finite element and level sets. Part II: level set update. International Journal for Numerical Methods in Engineering 2002; 53(11):2569-2586.

5. Belytschko T, Chen H, Jingxiao X, Goangseup Z. Dynamic crack propagation based on loss of hyperbolicity and a new discontinuous enrichment. International Journal for Numerical Methods in Engineering 2003; 58:1873-1905.

6. Duarte C, Hamzeh O, Liska T, Tworzydlo W. A generalized finite element method for the simulation of three-dimensional dynamic crack propagation. Computer Methods in Applied Mechanics and Engineering 2001; 190:2227-2262.

7. Réthoré J, Gravouil A, Combescure A. An energy conserving scheme for dynamic crack growth with the extended finite element method. International Journal for Numerical Methods in Engineering 2005; 63:631-659.

8. de Borst R, Remmers J, Needleman A. Mesh-independent numerical representations of cohesive-zone models. Engineering Fracture Mechanics 2006; 173(2):160-177.

9. Grégoire D, Maigre H, Réthoré J, Combescure A. Dynamic crack propagation under mixed-mode loadingcomparison between experiments and X-FEM simulations. International Journal of Solids and Structures 2007; 44(20):6517-6534.

10. Menouillard T, Réthoré J, Combescure A, Bung H. Efficient explicit time stepping for the extended finite element method. International Journal for Numerical Methods in Engineering 2006; 68(9):911-939.

11. Elguedj T, Gravouil A, Combescure A. Appropriate extended functions for X-FEM simulation of plastic fracture mechanics. Computer Methods in Applied Mechanics and Engineering 2006; 195(7-8):501-515.

12. de Borst R, Remmers J, Needleman A, Abellan M. Discrete vs smeared crack models for concrete fracture: bridging the gap. International Journal for Numerical and Analytical Methods in Geomechanics 2004; 28:583-607.

13. Rannou J, Gravouil A, Baïetto-Dubourg M. A local multigrid X-FEM strategy for 3-d crack propagation. International Journal for Numerical Methods in Engineering 2009; 77(4):581-600.

14. Gracie R, Oswald J, Belytschko T. On a new extended finite element method for dislocations: core enrichment and nonlinear formulation. Journal of the Mechanics and Physics of Solids 2008; 56:200-214.

15. Aubertin P, Réthoré J, de Borst R. Energy conservation of atomistic/continuum coupling. International Journal for Numerical Methods in Engineering 2009; 78(11):1365-1386.

16. Réthoré J, Hild F, Roux S. Extended digital image correlation with crack shape optimization. International Journal for Numerical Methods in Engineering 2008; 73(2):248-272.

17. Réthoré J, Hild F, Roux S. Shear-band capturing using a multiscale extended digital image correlation technique. Computer Methods in Applied Mechanics and Engineering 2007; 196(49-52):5016-5030.

18. Réthoré J, Besnard G, Vivier G, Hild F, Roux S. Experimental investigation of localized phenomena using digital image correlation. Philosophical Magazine 2008; 88(28-29):3339-3355.

19. Réthoré J, Tinnes J, Roux S, Buffière J, Hild F. Extended three-dimensional digital image correlation (X3D-DIC). Comptes Rendus Mécanique 2008; 336:643-649.

20. Nicaise S, Renard Y, Chahine E. Optimal convergence analysis for the extended finite element method. Available from: http://hal.archives-ouvertes.fr/hal-00339853, 2009.

21. Liu X, Xiao Q, Karihaloo B. XFEM for direct evaluation of mixed mode sifs in homogeneous and bi-materials. International Journal for Numerical Methods in Engineering 2004; 59:1103-1118.

22. Xiao Q, Karihaloo B. Direct evaluation of accurate coefficients of the linear elastic crack tip asymptotic field. Fatigue and Fracture of Engineering Materials and Structures 2003; 26(8):719-729.

23. Chahine E, Laborde P, Renard Y. Crack-tip enrichment in the XFEM method using a cut-off function. International Journal for Numerical Methods in Engineering 2008; 75(6):629-646.

24. Réthoré J, Roux S, Hild F. Noise-robust stress intensity factor determination from kinematic field measurements. Engineering Fracture Mechanics 2006; 75(13):3763-3781.

25. Roux S, Hild F. Stress intensity factor measurement from digital image correlation: post-processing and integrated approaches. International Journal of Fracture 2006; 140(1-4):141-157.

26. Chahine E, Laborde P, Renard Y. Spider-XFEM, an extended finite element variant for partially unknown crack-tip displacement. European Journal of Computational Mechanics 2008; 15(5-7):625-636. 


\section{HAX-FEM: A NEW ENRICHMENT PROCEDURE FOR CRACKED SOLIDS}

27. Williams M. On the stress distribution at the base of a stationary crack. ASME Journal of Applied Mechanics 1957; 24:109-114.

28. Ben Dhia H, Rateau G. The Arlequin method as a flexible engineering design tool. International Journal for Numerical Methods in Engineering 2005; 62:1442-1462.

29. Babuska I, Melenk J. The partition of unity method. International Journal for Numerical Methods in Engineering 1997; 40:727-758.

30. Xiao Q, Karihaloo B. Implementation of hybrid crack element on a general finite element mesh and in combination with XFEM. Computer Methods in Applied Mechanics and Engineering 2007; 196:1864-1873.

31. Bui H. Mécanique de la Rupture Fragile. Masson: Paris, France, 1978.

32. Karihaloo B, Xiao Q. Asymptotic fields at the tip of a cohesive crack. International Journal of Fracture 2008; 150:55-74. 